\title{
Charge exchange stripping cell measurements over a wide pressure range
}

\author{
R. J. Colchin
}

Oak Ridge National Laboratory, Oak Ridge, Tennessee 37831

\author{
A. L. Rcquemore and S. D. Scott
}

Princeton Plasma Physics Laboratory, Princeton University, Princeton, New Jersey 08544

The efficiency of the stripping cell incorporated in a charge exchange analyzer has been measured over a pressure range of 1.8 to $130 \mathrm{mtorr}$ and over an energy range of 1 to $130 \mathrm{keV} / \mathrm{amu}$. In these measurements, a beam of hydrogen atoms was ionized by helium gas. As a by-product of the measurements, the scattering cross section was also measured. At the lowest pressure, the scattering cross section compared well with those measured previously; at high pressure, the cross section no longer followed an energy power law. The analyzer efficiency (defined as the fraction of the atoms that is ionized and not lost through scattering) can be modeled over the entire range of energy and pressure by including the measured scattering cross sections.

\section{DISCLAIMER}

This report was prepared as an account of work sponsored by an agency of the United States Government. Neither the United States Government nor any agency thereof, nor any of their employees, makes any warranty, express or implied, or assumes any legal liability or responsibility for the accuracy, completeness, or usefulness of any information, apparatus, product, or process disclosed, or represents that its use would not infringe privately owned rights. Reference herein to any specific commercial product, process, or service by trade namc, trademark. manufacturer, or otherwise does not necessarily constitute or imply its endorsement, recommendation, or favoring by the United States Government or any agency thereof. The views and opinions of authors expressed herein do not necessarily state or reflect those of the 


\section{INTRODUCTION}

It is common practice to measure the ionization efficiency of the stripping cell in a neutral particle analyzer before using it as a plasma ion diagnostic. This calibration usually involves passing a beam of neutral particles through a stripping cell filled with hydrogen, helium, or nitrogen and measuring the ionized particle fraction. Scattering becomies a significant effect in the energy range of 100 to $5000 \mathrm{eV}$, which is of interest in many fusion experiments. Attenuation of the neutral flux by scattering in the stripping cell can significantly reduce the ionized efflux and is an important component of the calibration.

The work reported in this paper includes the measurement of ionization efficiency over wider pressure and energy ranges than are commonly used. The pressures used varied from 1.8 to 130 mtorr, and the energies were between 3 and $130 \mathrm{keV}$. A by-product of this effort is a measurement of the scattering cross section, which is compared with models in Sect. III.

\section{STRIPPING CELL MEASUREMENTS}

The stripping cell being calibrated is part of an E II $B$ type ch urge exchange analyzer, described previously by A. L. Roquemore et a!. ${ }^{1}$ A diagram of the calibration facility ${ }^{2}$ and analyzer is shown in Fig. 1 . The ion beam from the accelerator was neutralized in the drift chamber cell, and all residual ions were removed from the beam by the electrostatic deflection plates. The neutral beam then passed through the analyzer stripping cell chamber. Exiting particles were detected by a $25-\mathrm{mm}$-diam chevron-style microchannel plate located on the straight-through port. The analyzer stripping cell was $26 \mathrm{~cm}$ long with $1.5-\mathrm{mm}$ entrance and exit apertures. Helium gas was used in both the neutralizaticin and stripping cells. 
Since the scatiering, ionization, and neutralization processes are sensitive functions of pressure, it is important to calibrate the ionization gauge used to determine the stripping cell pressure against a standard. A wide range of pressure readings from a Variaña Bayert-Alpert style ion gauge was compared against a 1-torr MKS Baratron, ${ }^{b}$ which was used as the standard. A linear least squares fit was made to the data, which showed that the gauge factor varied between 4.33 (at 130 mtorr) and 6.56 (at 1.8 mtorr) as compared to the normally assumed gauge factor of 6.22 for helium. All pressures reported have been converted to true pressure by using the gauge factor.

The calibration procedure consisted of the measurement of the count rate: (1) with gas in the stripping cell and no applied magnetic field, (2) with gas in the stripping cell and a 2-kG deflection field applied, and (3) with no gas in the stripping cell (i.e., the incident count rate). The principal source of error in these measurements was beam noise, but counting statistics were adequate for pressures greater than 1.8 mtorr. Long-term drifts in the beam current were also present but contributed little error on the time scale required to take each data point.

This procedure yielded two independent bits of information. The difference between the count rates with the deflection magnet turned off and on divided by the incident count rate yielded the ionization efficiency (i.e., the fraction of the incident neutrals that is ionized and successfully strikes the channel plate without being lost through scattering). The fraction of the particles that is lost through scattering was obtained by subtracting the count rate with gas in the scattering cell from the incident count rate and dividing by the incident count rate. 


\section{EXPERIMENTAL RESULTS}

The angular scattering of a neutral or an ion moving along a straight line between two apertures located at either end of the stripping cell is characterized by a scattering cross section that is a funclion of both the energy and the scattering angle, $\sigma_{\text {scat }}=\sigma_{\text {scat }}(E, \theta)$. For the purpose of calibration, interest lies only in scattering events large enough to cause the particle to miss the exit aperture, thus removing it from the beam. Thus, the effective "removal"

scattering cross section $\left\langle\sigma_{\text {scat }}(E)\right\rangle$ is affected by both the angular scattering cross section and the ratio of the aperature diameter to the stripping cell length. The measured scattering fraction corresponding to $\left[1-\exp \left(-\left\langle\sigma_{\text {scat }}\right\rangle n I\right)\right]$ is plotted in Fig. 2 for pressures of 1.8 and 130 mtorr. Obtaining data at high pressure and low energy proved to be difficult because the loss caused by scattering was severe. Figure 2 shows least squares curves that have been fitted to the data. Such curves were used to generate the scattering cross sections.

Scattering cross sections measured at the pressure extremes of 1.8 and 130 mtorr are shown in Figs. 3 and 4. Data were also obtained at several intermediate pressures. The behavior varies with pressure, changing gradually from an (Energy) ${ }^{-x}$ power law behavior at low (1.8-mtorr) pressure to a more complicated behavior at high (130-mtorr) pressure.

The measured efficiencies for the same pressure extremes are shown in Figs. 5 and 6 . Scattering is relatively unimportant at energies above $15 \mathrm{keV}$ at the lower pressure of 1.8 mtorr, but it is still important up to $100 \mathrm{keV}$ at the higher pressure (130 mtorr). 


\section{DISCUSSION}

Previous measurements of proton scattering by helium gas tend to have been made at energies of a few $\mathrm{keV}$ and at pressures toward the low end of our range. The results ${ }^{3}$ typically fit $a \sigma_{\text {scat }}=a^{\star}\left(\right.$ Energy $^{-x}$ power law equation. Adlam and Aldcroft ${ }^{4}$ measured a to be $7.5 \times 10^{-16} \mathrm{~cm}^{-2}$ and $x=0.58$, while Amdur and Mason 5 found $x=0.58$ for a "narrow" detector and $x=0.64$ for $a$ "wide" detector (more nearly our case). Davis, Mueller, and Keane ${ }^{6}$ measured $a=9 \times 10^{-17}$ and $x=0.65$. A fit to our data at low pressure gives $a=1.57 \times 10^{-16}$ and $x=0.64$. However, a log-log plot of $\sigma_{\text {scat }}$ vs energy (see Fig. 4) shows that, as the pressure in the scattering cell is increased, a power law type of fit is no longer applicable. This is probably because of multiple scattering and the role played by the geometry of the scattering cell, since the scattering cross section itself is not pressure dependent. Particles will be scattered out of the incident beam, mainly by small angle scattering of both protons and neutrals. Differential small angle scattering cross sections have been measured for neutral scattering by Fleischmann, Barnett, and Ray ${ }^{7}$ and for both neutral and charged particle scattering by Sauers and Thomas. ${ }^{8}$ Small angle scattering scales as $\sigma_{\text {scat }} \approx \mathbf{k}[\mathbf{f}(\mathrm{s}) / \mathrm{E} \theta]^{2 / s}$, where Ruzic ${ }^{9}$ finds that $s=2.9, \theta$ is the angle for small angle scattering of neutrals, $f(s)=1.95, k=0.712 \mathrm{eV}-\dot{A}$, and $E$ is the energy in keV.

Calculations of the effective "removal" cross section have been made by using two models for the beam, one that assumes that the beam is a uniform solid cylinder and a second that models the beam as a line source along the center line of the ceil. We have made the unwarranted assumption that Ruzic's 
cross section is valid to energies of $150 \mathrm{keV}$, even though it was measured only to $=2 \mathrm{keV}$. The results of these two models are shown in Figs. 3 and 4 . At low pressure, the experimental results lie between results predicted by the two models (implying that the beam has a nonuniform profile). At high pressure, the measured scattering cross section equals that of the cylindrical model at low energy, but it falls off more steeply at high energy.

The efficiency $\eta$ for production of protons (which are not lost through scattering) is 4

$$
\eta=\frac{\sigma_{01}}{\sigma_{01}+\sigma_{10}}\left(1-\mathrm{e}^{-\left(\sigma_{01}+\sigma_{10}\right) n l}\right) \mathrm{e}^{-\left(\sigma_{-\infty}\right) n l},
$$

where $\sigma_{10}$ is the cross section for charge exchange and $\sigma_{01}$ is the cross section for ionization. Calculations were made by using the measured cross section for $\left\langle\sigma_{\text {scat }}\right\rangle$ as well as the line source model based on Ruzic's cross section. The results are shown in Figs. 5 and 6 . Although reasonable agreement with the data is obtained by using the measured and calculated scattering cross sections at low pressure, the results of the line source model differ (particularly at low energy) in the high-pressure regime

\section{ACKNOWLEDGMENTS}

This research was sponsored by the Office of Fusion Energy, U.S. Department of Energy, under Contract DE-AC05-840R21400 with Martin Marietta Energy Systems, Inc., and Contract DE-AC02-76CH03073 with Princeton University. 


\section{References}

aVarian Associates, Inc., 121 Hartwell Ave., Lexington, MA 02173

${ }^{b}$ MKS Instruments, Inc., 22-24 Third Avenue, Burlington, MA 01803.

${ }^{1}$ A. L. Roquemore, G. Gammel, G. W. Hammett, R. Kaita, and S.S. Medley, Rev. Sci. Instrum. 56 (5), 1120 (1985) .

2 A. L. Roquemore, R. J. Goldston, R. Kaita, and S. S. Medley, Bull. Am. Phys. Soc. $25(8), 950(1980)$.

${ }^{3}$ C. J. Armentrout, G. Bramson, and R. Evanko, E Parallel B Canted NeutralParticle Spectrometer, GA-A17688, GA Technologies, San Diego, Calif, 1985.

4.J. H. Adlam and D. A. Aldcroft, The Measurement of the Efficiency of a Helium Gas Cell for the Conversion of a Beam of Energetic Atomic Hydrogen into a Proton Beam, CLM-R100, 1969.

5 I. Amdur and E. A. Mason, J. Chem. Phys. 25 (4), 630 (1956).

${ }^{6}$ S. L. Davis, D. Mueller, and C. J. Keane, Rev. Sci. Instrum. 54 (3), 315 b (1983).

${ }^{7}$ H. H. Fleischmann, C. F. Barnett, and J. A. Ray, Phys. Rev. A 10 (2), 569 (1974).

${ }^{8}$ I. Sauers and E. W. Thomas, Phys. Rev. A 10 (3), 822 (1974).

${ }^{9}$ D. N. Ruzic, Total Scattering Cross-Sections and Interatomic Potentials for Neutral Hydrogen and Helium on Some Noble Gasses, Ph.D. Thesis, Princeton University, Princeton, N.J., 1984. 


\section{Figure Captions}

FIG. 1. Schematic of the calibration iacility, including an ion source, the drift chamber, and the $E_{\|} B$ analyzer.

FIG. 2. Fraction of ions scattered vs energy.

FIG. 3. Total scattering cross section for $\mathrm{H}$ scattering on $\mathrm{He}$ vs energy at a pressure of 1.8 mtorr.

FIG. 4. Total scattering cross section for $\mathrm{H}$ scattering on He vs energy at a pressure of 130 mtorr.

FIG. 5. Efficiency $\eta$ for ionization of $H^{0}$ by $\mathrm{He}$ at a pressure of $1.8 \mathrm{mtorr}$.

FIG. 6. Efficiency $\eta$ for ionization of $\mathrm{H}^{0}$ by $\mathrm{He}$ at a pressure of $130 \mathrm{mtorr}$. 


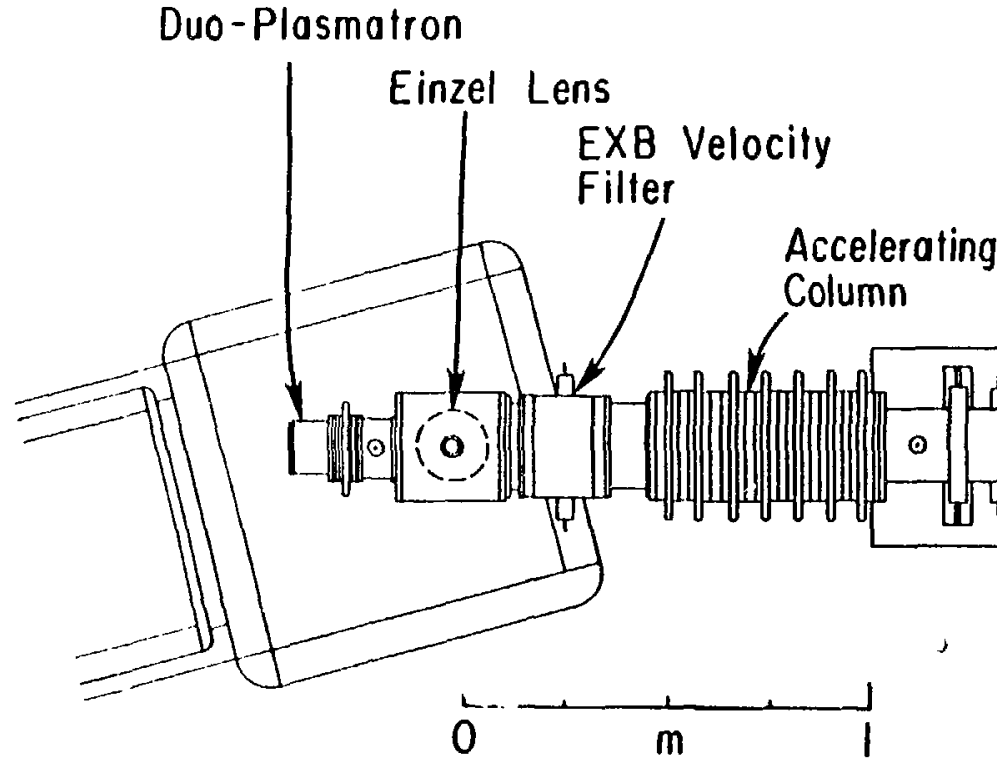

ION SOURCE
EIIB ANALYZER 




点 


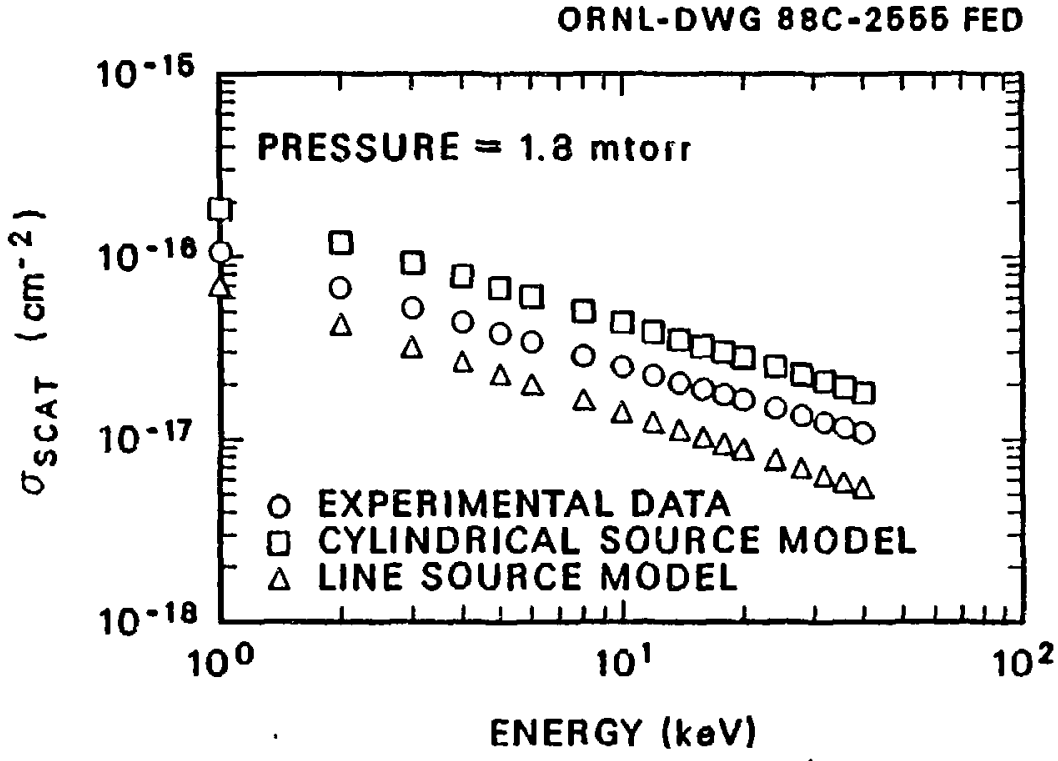

FIGURE 3 
ORNL-DWG 88C-2566 FED

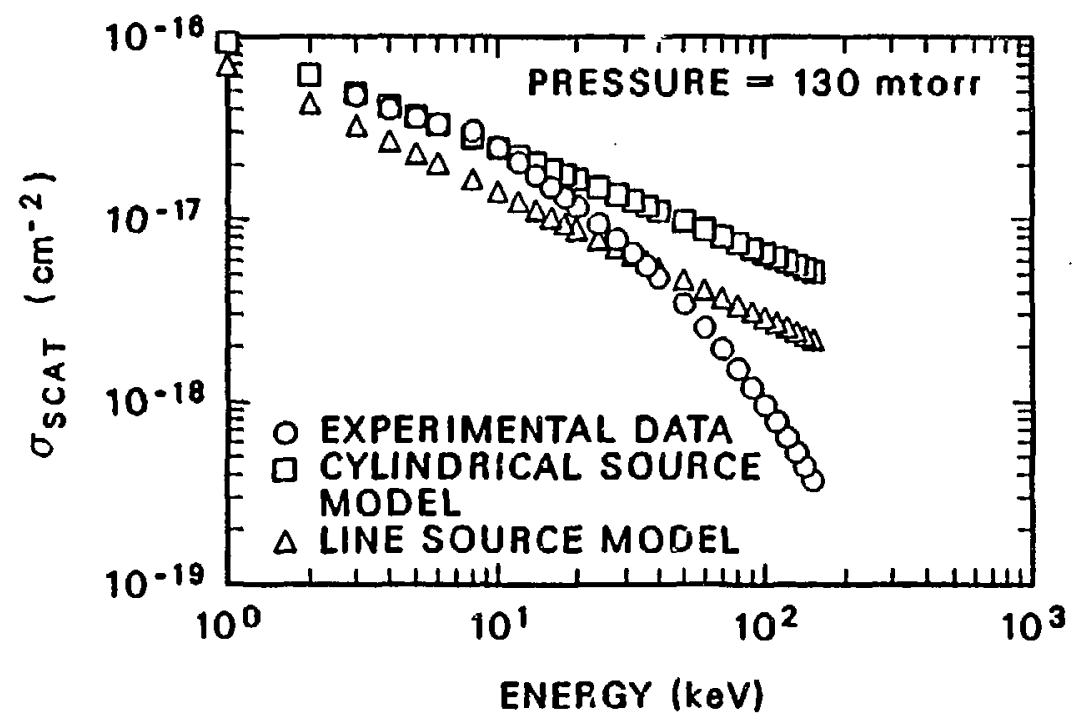

FIGURE 4 


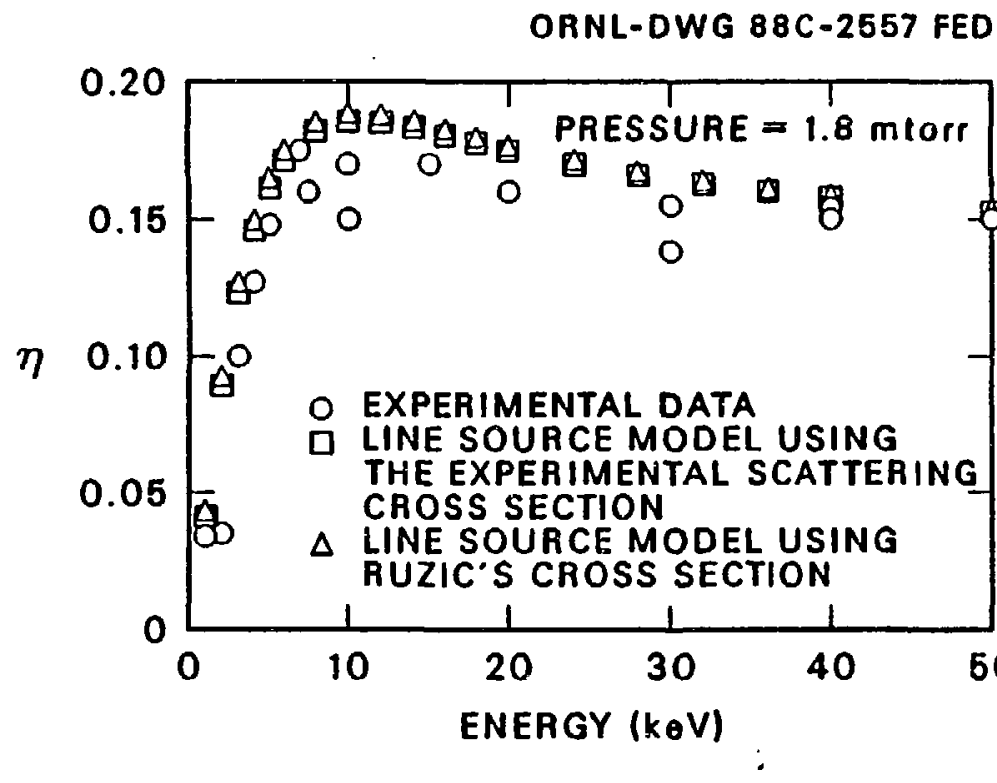

FIGIJRE 5 


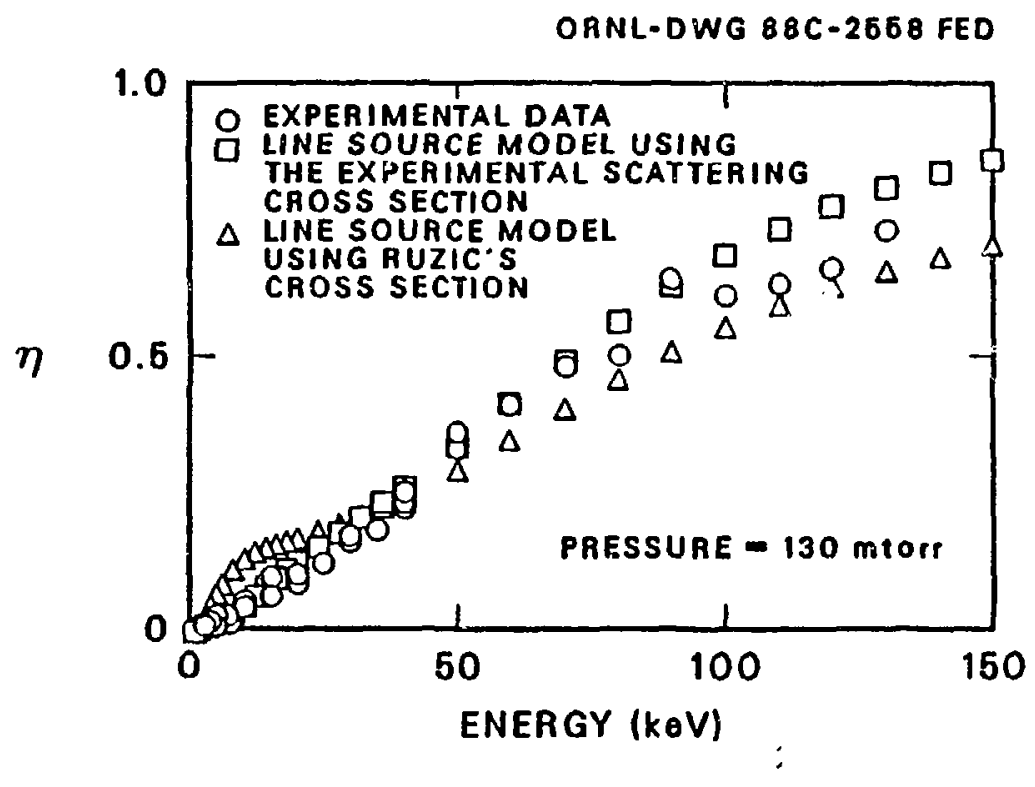

FIGURE 6 